STUDI

FRANCESI

\section{Studi Francesi}

Rivista quadrimestrale fondata da Franco Simone

159 (LIII | III) | 2009

Varia

\title{
L'Image du Nord chez Stendhal et les Romantiques, textes réunis par Kajsa Andersson
}

\author{
Michel Arrous
}

\section{(2) OpenEdition}

1 Journals

\section{Édition électronique}

URL : http://journals.openedition.org/studifrancesi/7593

DOI : 10.4000/studifrancesi.7593

ISSN : 2421-5856

Éditeur

Rosenberg \& Sellier

\section{Édition imprimée}

Date de publication : 1 décembre 2009

Pagination : 632-635

ISSN : 0039-2944

\section{Référence électronique}

Michel Arrous, "L'Image du Nord chez Stendhal et les Romantiques, textes réunis par Kajsa Andersson », Studi Francesi [En ligne], 159 (LIII | III) | 2009, mis en ligne le 30 novembre 2015, consulté le 09 janvier 2021. URL : http://journals.openedition.org/studifrancesi/7593 ; DOI : https://doi.org/10.4000/ studifrancesi.7593

Ce document a été généré automatiquement le 9 janvier 2021.

\section{(c) $($ ) $\odot$}

Studi Francesi è distribuita con Licenza Creative Commons Attribuzione - Non commerciale - Non opere derivate 4.0 Internazionale. 


\title{
L'Image du Nord chez Stendhal et les Romantiques, textes réunis par Kajsa Andersson
}

\author{
Michel Arrous
}

\section{RÉFÉRENCE}

AA. vV. L'Image du Nord chez Stendhal et les Romantiques, textes réunis par Kajsa ANDERSSON, Örebro University, University Library, t. III, 2006, pp. 465; t. IV, 2007, pp. 470.

1 Ces deux volumes prolongent heureusement la tâche entreprise par Kajsa Andersson avec la publication des actes du colloque international d'Örebro, en 2002, consacré aux rapports de Stendhal et des romantiques avec l'Europe du Nord. Vingt-sept études dans le tome III, vingt-cinq dans le tome IV: de cette abondance, on ne saurait rendre compte dans le détail! Sont convoqués Andersen, Mme de Staël, Xavier de Maistre, Stendhal, Mérimée, Balzac, Hugo, Sand, Michelet, Tocqueville, Dumas, Marceline DesbordesValmore, Pétrus Borel, Baudelaire, Flaubert, Claudel, mais aussi Désirée Clary, pour ses lettres, et quelques voyageurs. Le maître d'œuvre, K. ANDERSSON, a signé deux contributions au culte valmorien: dans le t. III, La rencontre des cultures dans 'L'Atelier d'un peintre" (pp.12-39), roman à l'évidente dimension autobiographique, publié en 1833 (réédité par Aragon en 1949), et, dans le t. IV, La Pologne dans la vie et l'œuvre de Marceline Desbordes-Valmore (pp.45-71). La première est consacrée à l'accueil malheureusement fort modeste que les manuels d'histoire littéraire ont réservé à l'auteur des Roses de Saadi ou de La Couronne effeuillée. Son nom n'est peut-être plus «qu'une ombre douce et vaine», ce qu'il n'était assurément pas pour Baudelaire, Anatole France ou Proust, ni pour les générations d'écoliers qui ont récité: «J'ai voulu ce matin te rapporter des roses...»! La seconde illustre, si besoin était, l'amour marcelinien toujours attentif aux souffrances d'autrui. Cette fois Marceline, se penchant sur les réalités de la vie dans leur aspect politique, se tourne vers la Pologne, pays qu'elle n'a jamais visité. Elle milite dans la section lyonnaise du «Bazar polonais» 
et, porte-parole d'une Europe libre et unifiée, elle adapte habilement en 1831 et 1839 deux poèmes lyriques de Jozef Zaleski (1802-1886) et publie deux contes pour enfants en 1865 .

Voici un aperçu du tome III. C'est l'imaginaire de la Russie, seconde patrie de Xavier de Maistre, que Xavier BOURDENET (pp. 105-127) analyse dans Les Prisonniers du Caucase et La Jeune Sibérienne (1825), deux nouvelles sous le signe de l'ambivalence idéologique. Dans Stendhal et l'Europe du Nord (pp. 201-218), modestement sous-titré Sur quelques souvenirs de 1812, Francis CLAUDON apporte non seulement d'utiles compléments, mais aussi corrige quelques approximations fort reçues sur le mythe du Nord construit par Herder et Mme de Staël. Stendhal s'était renseigné sur ces contrées avant de les parcourir en lisant l'abbé Georgel et Rulhière. Bien qu'il n'ait laissé aucune relation d'ensemble, de ses rapides digressions et de ses allusions, il ressort qu'il a vécu le Nord comme la découverte de l'Autre, un Nord éprouvé dans sa réalité concrète comme un tout (le vent, le froid et la boue, les Cosaques incarnation de la férocité), comme le monde de la sauvagerie totale. Nicolas BOUSSARD confronte Les voyages et les philosophies parallèles de Staël et de Stendhal (pp. 128-155) en retraçant précisément les itinéraires de ces deux exilés en désaccord sur bien des points, entre autres la politique de Napoléon face à la Russie. Deux visions des territoires ravagés du despotisme, deux romantismes différents: l'exilée involontaire a une vision poétique, le volontaire, le «Déplanté», jure de ne plus s'éloigner de la «cara Italia», mais tous deux aboutissent au même constat: les Russes sont certes les victimes du despotisme, mais sont animés d'une énergie qui en fait «les hommes les plus énergiques dont l'histoire fasse mention» (Histoire de la peinture en Italie), une énergie physique et patriotique (avec gallophobie). Mme de Staël, qui n'a assisté à aucun combat de la campagne, se livre à une étonnante apologie de la guerre; Stendhal, qui a assisté et participé aux combats de la retraite, n'a plus aucune illusion. Les réconcilie provisoirement leur semblable réaction face à l'invasion et à l'occupation de la France par les Cosaques d'Alexandre. En comparant deux textes, la très dogmatique Érotique comparée de Charles de Villers (1806) et De l'Amour, le premier reposant sur l'opposition France/Allemagne, le second sur l'opposition Nord/Sud, JeanJacques HAMм (pp. 242-254) illustre l'antinomie au cœur du colloque d'Örebro. Bien que les corpus soient assez proches, les démarches sont foncièrement différentes car leur origine, historique pour le premier dont les choix idéologiques et géographiques sont sans ambiguïté, personnelle pour le second qui relève la fragilité ou l'instabilité de la vérité psychologique, la tension existe entre vérité générale assénée dogmatiquement et vérités locales.

3 Homme de la rencontre des cultures, Mérimée offre bien sûr un vaste terrain d'étude. Janine GALLANT, dans "Colomba" ou le vertige de la rencontre (pp. 230-241), étudie les stratégies narratives qui, contrairement à une opinion répandue, ne contribuent pas à opposer nettement deux civilisations: intrigue hésitante voire opaque, points de vue multiples, ambiguïté des personnages, oscillation entre le tragique et l'humoristique, narrateur tantôt omniprésent, tantôt défaillant. Mérimée ne tranchant jamais «entre répulsion et inclusion de l'étrangeté», le lecteur reste dans l'incertitude. Thierry ozWALD (Le Nord de Mérimée: un horizon plombé, pp. 353-366) a, quant à lui, privilégié les rares œuvres qui mettent en jeu «l'inclination septentrionale de la rêverie mériméenne» - Les Espagnols en Danemark, Vision de Charles XI et Lokis - pour montrer que leur problématique a, par bien des points, une étroite parenté avec l'exotisme du Sud. Ainsi, Lokis pourrait être lue comme une version nordique de Carmen. Les 
«nouvelles du Nord», plus énigmatiques et plus sauvages que les autres, mettraient en scène l'univers névrotique de Mérimée. Si l'exercice qui consiste à transposer du Nord au Sud ne manque pas d'intérêt, dans la mesure où il révélerait «un canevas toujours identique», on peut s'interroger sur la légitimité du recours à la notion fort ambiguë d'exotisme, ou plus exactement à la «quête d'exotisme» qui caractériserait la démarche mériméenne, à moins que ce ne soit avec son souci propre à lutter contre l'uniformisation en marche. Plus stimulante est l'image du Nord qu'on perçoit dans Lokis et Charles XI, comme «un théâtre de passions où se joue sans cesse la réalisation illusoire d'un désir frustré». La recherche de matériaux psychanalytiques qu'une rapide archéologie révèle permet à Th. Ozwald, particulièrement dans Lokis, de mener à bien son analyse d'un scénario archaïque.

L'opposition Nord/Sud telle que l'avaient formulée Bonstetten et Mme de Staël se retrouve dans Consuelo à travers l'opposition des lieux et des personnes, particulièrement entre Albert, le génie du Nord, mais sans corps, et Anzoletto, la vie méridionale, mais sans âme. Pour Catherine MARIETTE-CLOT (pp. 221-229) cette opposition, en forme de dilemme amoureux, révèle une préoccupation anthropologique qui affirme la possibilité d'un équilibre entre le Nord et le Sud. Pour quelles raisons George Sand a-t-elle fait le choix de la Dalécarlie pour L'Homme de neige (1859)? Après Joseph-Marc Bailbé et Brigitta Berglund-Nilsson (t. I, pp. 33-40; t. II, pp. 33-43), Martine REID (Aux sources de "L'Homme de neige": la Suède livresque de George Sand, pp. 368-381) reprend ici l'étude de ce roman qu'on a longtemps négligé. Une lecture attentive du «feuilleté intertextuel» démontre que Sand, persuadée des nécessités de l'illusion référentielle, s'est sérieusement documentée en lisant récits de voyage, comptes rendus d'expéditions, ouvrages de vulgarisation, mais aussi Les Mines de Falun d'Hoffmann et, bien sûr, les récits de Xavier Marmier. Yvon LE SCANFF illustre La fécondité de l'opposition Nord/Sud dans l'esthétique sandienne (pp. 306-319): chez la romancière, cette opposition génère un subtil réseau tissé par l'imaginaire, l'esthétique et l'idéologie, aussi bien dans L'Homme de neige que dans Mouny-Robin, conte berrichon fantastique (1869).

5 Le tome IV est tout aussi riche que le précédent. Annika ALLING-MÖRTE (La réception de Stendhal en Suède, pp. 12-42) évoque la réaction positive des premiers lecteurs suédois de Stendhal qui n'ont pas attendu la traduction de la Chartreuse (1902) puisque c'est en 1830, l'année même de sa publication, qu'est traduit Lord Byron en Italie». Tout aussi révélatrice est la présence de Stendhal dans les encyclopédies de 1905 à 1930. Le point de vue des critiques et historiens suédois subit une inflexion nettement négative dans les années 1920 (absence d'originalité et d'imagination), et ce jusque dans les années 50 où se forme une nouvelle vague Stendhal, faite de nombreuses traductions et d'articles savants. La grande vague arrive dans les années ' 90 où Stendhal concurrence Balzac, années pendant lesquelles les traductions continuent (Armance, Lucien Leuwen; des romans, il ne reste qu'à traduire Lamiel). Depuis le début du nouveau millénaire, on note une sensible décrue des études stendhaliennes. Pour sa part, Merete NIELSENGERLACH (pp. 271-284) apporte des précisions sur quelques points qu'elle a déjà évoqués quant à la réception de Stendhal au Danemark et en Norvège où un renouveau stendhalien se manifeste depuis 1995, et en Suède, avec l'exemple du critique Horace Engdahl, né en 1948. Autre étude de réception, les Métamorphoses de Baudelaire - la réception en Suède de Christina SJöBLAD (pp. 384-408) qui fixe l'image du poète jusque dans les années 1900, laissant de côté son influence sur les auteurs suédois. 
Le pays des chimères est-il au Nord? se demande Marie-Rose CORREDOR (pp. 91-97) à propos d'Armance et de Mina de Vanghel, femmes à imagination dont l'imagination divague vers cet Ailleurs. Partant de l'opposition classique entre matérialisme et idéalisme, Michel CROUZET (Le Nord, le Midi: de l'aisthesis à l'esthétique, pp. 99-108) étudie les rapports de l'homme romantique, l'homme-artiste, avec les choses, avec la «chair du monde» (Merleau-Ponty), un homme qui «connaît mieux le monde que la scientificité et la technicité réunies». C'est la sensibilité au monde sous ses deux versants, le Nord et le Midi, qui est en cause, chacun avec sa mélancolie propre: au Nord, «la vie est inférieure à l'âme»; au Midi, «l'âme se découvre inférieure à la vie». Dans le cas du Sud, la mélancolie telle que la comprend Stendhal est érotique. Brève et dense leçon sur «le savoir romantique» à la lumière de la phénoménologie. Stendhal romancier apporte-t-il du nouveau dans la représentation de l'amour et raconte-t-il l'amour d'une manière réaliste ou romantique? Double question à laquelle Björn LARSsON (pp. 256-269) essaie de répondre en relevant, à l'aide de quelques exemples comme l'amour-passion à l'épreuve du doute ou bien le rôle ou la place de la sexualité, les gauchissements que Stendhal fait subir à la tradition qui va de Tristan et Yseut au Lys dans la vallée. Pour évoquer Stendhal, son époque et l"'Athènes de l'Allemagne" (pp. 328-352), Elisabeth SCHEELE propose une revue exhaustive des affinités entre Stendhal et le classicisme allemand.

Béatrice DIDIER (pp.111-123) rappelle l'influence considérable qu'eut De l'Allemagne sur le romantisme français, en dépit de ses lacunes. Les chapitres consacrés aux écrivains, surtout «De la poésie classique et de la poésie romantique», constituent un manifeste dans lequel il faut voir «un art poétique du romantisme à venir». B. Didier relève que l'accueil réservé au livre fut beaucoup plus favorable en France qu'en Allemagne où Heine reprocha à Mme de Staël d'avoir créé par ses présupposés ce qu'il faut bien appeler le mythe de l'Allemagne.

8 Dans Image ou mirage du Nord? Autour du "Fou du roi de Suède" de Pétrus Borel (pp.125-144), Barbara Dimopoulou étudie cette œuvre peu connue qui est en fait une traduction-adaptation d'une nouvelle de Mary Shelley plutôt qu'un plagiat. Dans son exercice de réécriture, Pétrus Borel a transposé le lieu de l'action de l'Italie en Suède et du Moyen Âge à l'époque contemporaine, faisant d'un texte faussement suédois une œuvre faussement autobiographique dans une France démoniaque, alors que la Suède est présentée comme une terre d'élection.

$9 \quad$ La spécificité nordique est sans doute plus sensible dans le Voyage en Russie de Dumas qui, pour une fois, abandonne l'univers méditerranéen. Sylviane EPPSTEIN-ROBARDEY (Le Nord vu par Dumas père dans Voyage en Russie - Entre critique et cliché: rhétorique de l'équivalence et poétique de l'unicité, pp.169-188) s'est limitée aux pages consacrées à Saint-Pétersbourg dont les nuits sont loin d'égaler celles de Naples, Tunis ou Alger, à la Finlande, pays nébuleux, et à quelques passages évoquant la Suède. Dumas voudrait fixer une certaine essence nordique en l'opposant au Sud toujours présent dans son texte; il se montre attentif aux légendes, aux comportements culturels et même aux spécificités idiomatiques. Son Nord est insolite, exceptionnel («Rien ne se fait ici comme ailleurs»).

10 L'Homme qui rit, «au cœur de l'univers hugolien» (P. Albouy), est une tentative longtemps méconnue de marier tragédie et comédie dans l'esprit de Shakespeare. Hans-Roland JOHNSON (pp. 205-225) revient sur la pratique et le fonctionnement de l'antithèse dans ce roman du Nord dont le texte se présente comme un véritable carrefour d'énoncés, une tentative aboutie d'égaler Shakespeare. Après l'Angleterre, la 
Norvège dans Han d'Islande: François KERLOUÉGAN (pp. 227-238) isole un aspect négligé de ce roman noir exotique, le paysage nordique. Plus que le dépaysement, importe la construction d'un cadre romanesque investi par l'imaginaire. La Norvège hugolienne devient l'espace du songe et de la vie méditative.

De Séraphîta, habituellement considéré comme un havre de paix dans l'œuvre de Balzac, Pierre LAFORGUE ("Séraphîta", ou la révolution chez les anges, pp. 241-255) propose une lecture nouvelle d'orientation sociocritique. Contrairement à ce qu'on a pu en dire, ce roman trop souvent considéré comme un poème ou une divagation mystique (M. Bardèche) a toute sa place dans l'œuvre de Balzac. Remarquant l'absence de tout tropisme scandinave chez Balzac et interrogeant l'ensemble du paradigme norvégien, P. Laforgue montre, d'une part, que la signification symbolique du paysage fait de ce pays un lieu abstrait, un lieu de méditation où se développent des conceptions mystiques et spiritualistes, et, d'autre part, que cet espace de neige et de glace «est aussi un espace géo-politique» à déchiffrer à la lumière de la problématique du Nord et de l'Orient. Le personnage de Wilfrid, véritable frère de René, est au centre de ce roman dont on propose une captivante «lecture bonapartiste». Mais l'aventure de Wilfrid a échoué, alors que Séraphîta est l'ange d'une révolution spirituelle dont Swedenborg est le prophète. Cette nouvelle lecture permet de comprendre que ce roman ne peut être séparé d'autres œuvres contemporaines, par exemple Le Contrat de mariage et Le Lys dans la vallée.

12 Le lien entre religion et politique n'est pas perdu de vue avec les Légendes démocratiques $d u$ Nord. À partir de la dichotomie Nord/Sud sur laquelle se fondait une nouvelle littérature (Mme de Staël, Schlegel), Gisèle SEGINGER (Géographie et idéologie: Michelet et les "Légendes démocratiques du Nord", pp.355-371) retrace l'invention d'une géographie paradoxale. Pour Michelet, la Russie, "géant ivre et fou», dans une Europe tournée vers le Nord, est du Sud, et la Pologne du Nord. D'où la nécessité de lutter contre la Russie pour aider la Pologne, cette seconde France plus jeune, «avec tous nos anciens défauts, nos qualités, mais le martyre de plus...». Dans une explication para-scientifique où sociologie et physiologie se rejoignent, les Russes sont un peuple méridional de race et d'esprit qui se trouve malheureusement exilé au Nord. Ils sont une race affaiblie, énervée, incapable de volonté et de liberté. La Russie, sans véritable État - une nation ne peut exister que si elle a un centre - est une allégorie du Mal.

Détour inattendu jusqu'aux "extrêmes limites de la civilisation européenne», en compagnie de Tocqueville et de Gustave de Beaumont dans leur bref voyage de 1831 chez les Indiens du Michigan, ou plutôt sur les traces des pionniers appliquant leur morale de l'utilitas, et bourreaux des nobles sauvages. C'est ce récit chateaubrianesque, du moins aux yeux des premiers lecteurs de Quinze Jours dans le désert, texte posthume, que Francesco SPANDRI (L'héroïsme sans vertu du pionnier tocquevillien, pp.425-437) présente comme un exemple un peu inattendu de la dichotomie en question: le pionnier prosaïque, «partisan de l'utile», benthamien sans le savoir, incarne l'esprit nordique.

14 Mérimée, homme du Midi et Grec s'il en fut, est lui aussi au rendez-vous. Hans FARNLOF aborde la question de La mythification dans "Colomba" (pp. 191-203) en se demandant comment l'écrivain exploite les lieux communs, la «couleur locale». Le récit subit une «ironisation» permanente qui dénonce le regard porté sur l'autre. Dans l'étude qu'elle a consacrée à Quelques images du Nord et du Midi dans l'œuvre de Mérimée (pp. 299-326), Clarisse REQUENA évoque dans le détail les images du Nord et du Midi dans l'œuvre de 
Mérimée. Certes, l'ethnologie de ce cosmopolite tantôt Cosaque, Gitan, Moldo-Valaque, Corse ou Français du XVI ${ }^{e}$ siècle est incomplète: Mérimée n'a pas évoqué la Scandinavie ou le grand Nord de l'Europe, et son Danemark n'est que l'antithèse de l'Espagne, sa patrie morale et littéraire, bien qu'il soit Anglais d'humeur et de goût! Plutôt que d'une polarisation Nord/Sud, il faudrait parler chez Mérimée de l'opposition sauvage/civilisé. Comme bien des romantiques, Mérimée juge le Nord de l'Europe (Nord français, anglais, allemand) plus civilisé que le Sud plus sauvage, un Sud animal - qu'on pense à Mateo Falcone, Colomba, Carmen - opposé au froid et raisonnable Nord, monde de la cruauté civilisée.

Parmi les voyageurs français en Scandinavie, il y en eut de bien moins célèbres que Xavier Marmier, mais qui méritent cependant qu'on les tire de l'oubli, entre autres Louis-Antoine Léouzon-Leduc (1815-1889) dont Marthe PEYRoux (pp. 287-296) a eu l'excellente idée d'évoquer les voyages dans les «pays du Nord», mais il aurait fallu rappeler son œuvre de traducteur du Kalévala qui inspira Lecomte de Lisle et même Dumas, ainsi que ses voyages et missions en Russie.

Étudiants et spécialistes trouveront dans ces deux volumes bien des aperçus variés et heureux sur le mythe du Nord en France et en Europe. Force est de constater que la plupart des romantiques ne se sont pas laissés prendre par les trop célèbres brumes du Nord et que fort rares ont été ceux qui se sont perdus dans de «fumeuses rêveries nordiques» comme ce fut le cas au siècle dernier, ainsi que le rappelle Régis Boyer dont il convient de citer cette définition: «le Nord, c'est l'ailleurs et l'autrement». 